7th International Workshop on Astronomy and

Relativistic Astrophysics (IWARA 2016)

International Journal of Modern Physics: Conference Series

Vol. 45 (2017) 1760020 (5 pages)

(C) The Author(s)

DOI: $10.1142 / \mathrm{S} 2010194517600205$

\title{
Magnetic Suspension with Motorization to Measure the Speed of Gravity
}

\author{
Henrique Linares, Carlos Frajuca, Fabio S. Bortoli, Givanildo A. Santos, \\ and Francisco Y. Nakamoto \\ Departamento de Mecânica \\ Instituto Federal de Educação, Ciência e Tecnologia de São Paulo (IFSP) \\ Rua Pedro Vicente, 625, 01109-010 São Paulo, Brazil \\ frajuca@ifsp.edu.br,bortoli.fabio@gmail.com,givanildo@ifsp.edu.br,nakamoto@ifsp.edu.br
}

Published 15 August 2017

\begin{abstract}
This work aims to design a magnetic suspension for an experiment to measure gravitys velocity. Such device must rotate two objects symmetrically with the greatest mass and largest radius as possible, at the speed of $500000 \mathrm{rpm}$, which means this device falls into the high-speed machines category. The guidelines and solutions proposed in this paper constitute a contribution to this class of engineering problems and were based on an extensive literature search, contacts with experts, the tutors and author's experience, as well as on experimental results. The main solution proposed is a hybrid bearing that combines a radial passive magnetic bearing with an axial sliding bearing, here called MPS (Magnetic Passive and Sliding) bearing.
\end{abstract}

Keywords: Gravity Speed, Ultrahigh Speed, Control, Vacuum, Magnetic Bearing.

PACS numbers: 04.30.Db and 04.80.Cc

\section{Introduction}

The speed of gravity is one of the great mysteries of nature. One way to measure the speed of gravity would be through the detection of gravitational waves but this is very difficult because until today only two gravitational wave events were detected. Besides the detection of such wave, a light counterpart must be found at the same time with different instruments, which makes this option almost impossible. The better option to achieve that goal is to produce a signal and detect it. In order to achieve this, it is proposed to develop a rotating system running at 600,000 rpm (rotations per minute) (theoretical limit is 2,000,000 rpm), and measure the signal at some distance. This presents great challenge in the parts of magnetic suspension, direct drive, control of rotating systems and highly resistant materials.

This is an Open Access article published by World Scientific Publishing Company. It is distributed under the terms of the Creative Commons Attribution 4.0 (CC-BY) License. Further distribution of this work is permitted, provided the original work is properly cited. 


\section{H. Linares et al.}

\section{Theoretical Foundation}

The velocity of gravitational waves in the theory of general relativity equals the velocity of light in vacuum, $c$ (see Ref. ${ }^{1}$ ). Within the theory of special relativity, the constant $c$ is not exclusively about light; on the contrary, it is the highest possible speed. Formally, $c$ is a conversion factor for changing the unit of time to the unit of space. $^{2}$

Before this, Newtonian theory of gravity required that the speed of gravity was infinite. This hypothesis was adequate to explain all the phenomena with the accuracy of observation of the time. Until the 19th century only an anomaly in astronomical observations could not be reconciled with the Newtonian gravitational model of instantaneous action: the French astronomer Urbain Le Verrier determined in 1859 that the elliptical orbit of the planet Mercury precessed at a rate significantly different from that predicted by the Newtonian theory. ${ }^{3}$

The first attempt to combine a finite gravitational velocity with Newton's theory was made by Laplace in 1805 . He considered a model in which the gravitational field is defined as a field of radiation or a fluid. Changes in the movement of the capture body are transmitted by some type of waves. ${ }^{4}$ Therefore, the motions of the celestial bodies must be modified in the order $v / c$, where $v$ is the relative velocity between bodies and $c$ is the speed of gravity. The effect of a finite velocity of gravity goes to zero as $c$ goes to infinity, but not as $1 / c^{2}$ as it does in modern theories. This led Laplace to conclude that the velocity of gravitational interactions is at least $7 * 10^{6}$ times the speed of light.

In 1900 Hendrik Lorentz attempted to explain gravity based on his ether theory and Maxwell's equations. The resulting net force is exactly what is known as universal gravity, in which the speed of gravity is that of light. This leads to a conflict with the law of gravity of Isaac Newton in which it was demonstrated by Pierre Simon Laplace that a finite velocity of gravity leads to some kind of aberration and therefore causes the orbits to be unstable. However, Lorentz showed that the theory is not covered by Laplace's critique because of the structure of Maxwell's equations: only effects in the order $v^{2} / c^{2}$ arise. But Lorentz calculated that the value for Mercury's advance perihelion was very low. ${ }^{5}$

In 1908, Henri Poincaré examined Lorentz's gravitational theory and classified it as compatible with the principle of relativity, but (as Lorentz) criticized the imprecise indication of the advance of the perihelion of Mercury. ${ }^{6}$

The finite velocity of gravitational interaction in general relativity does not lead to types of problems such as the aberration of gravity that Newton was originally concerned because there is no such aberration in static field effects. ${ }^{7}$

The first direct observation of gravitational waves, from the fusion of a pair of black holes, on September 14, 2015 (announced by the LIGO and Virgo collaborations on February 11, 2016 (see Refs. ${ }^{8-10}$ )) allowed a more direct measurement of its speed. Another attempts are made to detect gravitational waves ${ }^{11-17}$. 


\section{This Experiment}

Therefore, to directly measure the velocity of gravity, a tidal gravitational signal emitter and a detector are proposed to receive this signal. The speed will be obtained by the phase difference between the two devices weighted by distance. Since the speed of this signal must be very high, the device must rotate at a very high speed.

\section{Metodology}

Rotating Quadriple mass system can be seen on Fig. 1. The magnetic suspension can be seen on Fig. 2 and, as the driver device a variable magnetic reluctance motor was chosen and can be seen on Fig. 3.

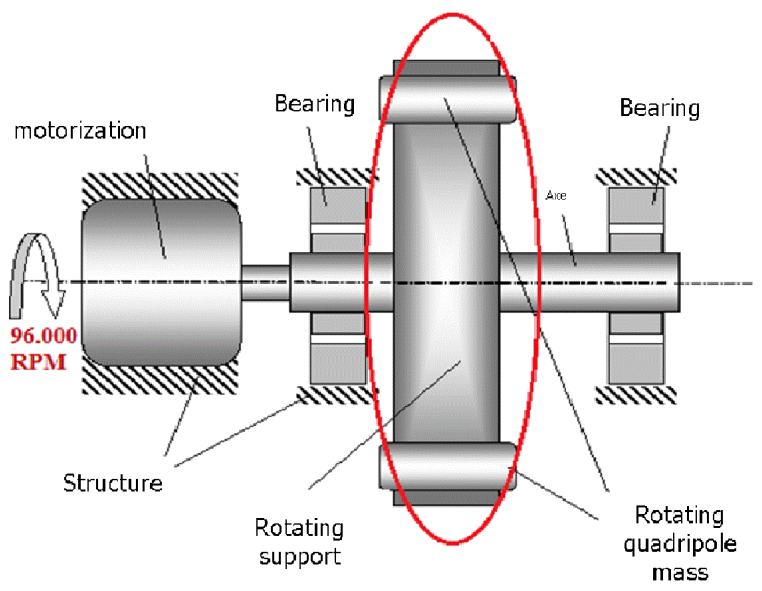

Fig. 1. The detailed gravitational signal generator.

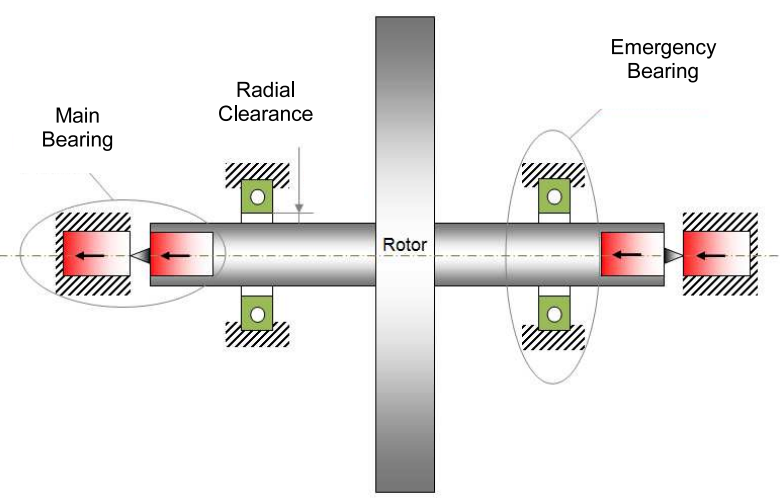

Fig. 2. The suspension device. 
H. Linares et al.

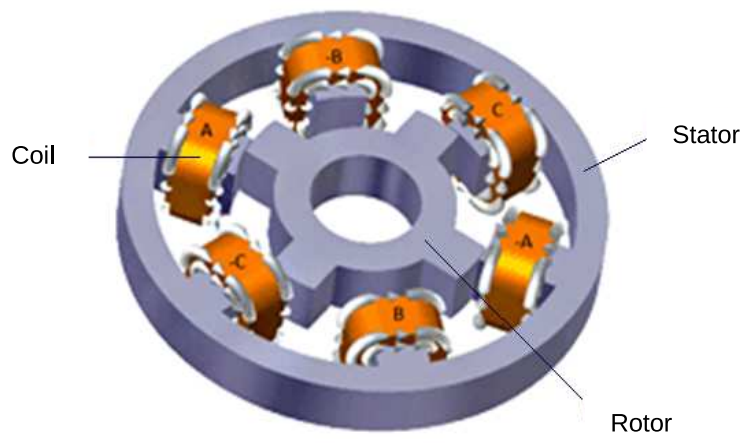

Fig. 3. The driver device.

\section{Conclusion}

There are few more steps to advance the design but we are confident that, with this design, a rotation of $500000 \mathrm{rpm}$ can be achieved, allowing measurable signal in the detector in a distance of 1 kilometer.

\section{References}

1. J. B. Hartle, Gravity: An Introduction to Einstein's General Relativity. (AddisonWesley, Boston, 2003) ISBN 981-02-2749-3, p. 332.

2. U. Le Verrier Lettre de M. Le Verrier à M. Faye Sur la Thorie de Mercure et Sur le Mouvement du Périhélie de Cette Planète, C. R. Acad. Sci. 49, 379 (1859).

3. P. S. Laplace, A Treatise in Celestial Mechanics, Vol. IV, Book X, Chapter VII (1805), translated by N. Bowditch (Chelsea, New York, 1966).

4. J. Zenneck, Gravitation, Encyklopädie der Mathematischen Wissenschaften mit Einschlussihrer Anwendungen (in German), (doi: 10.1007/978 - 3-663-16016 - 8_2) Vol. 5, (1903), p. 25.

5. H. A. Lorentz, Considerations on Gravitation, Proc. Acad. Amsterdam 2, 559 (1900).

6. H. Poincaré La dynamique de L'électron (PDF). Revue Générale des Sciences Pures et Appliqués 19386 (1908). Reprinted in Poincaré, Oeuvres Vol. IX, S. 551 and in Science and Method, (1908).

7. S. Carlip Aberration and the Speed of Gravity, Phys. Lett. A 267 (23), 81 ( 2000). (arXiv:gr-qc/9909087)(1999).

8. LIGO Scientific Collab. and Virgo Collab. (B. P. Abbott et al.), Phys. Rev. Lett., 116 (6), 061102 (2016). Observation of Gravitational Waves from a Binary Black Hole Merger. arXiv:1602.03837(2016), doi:10.1103/PhysRevLett.116.061102 (PDF).

9. D. Castelvecchi and A. Witze. Einstein's Gravitational Waves Found at Last, Nature News, doi:10.1038/nature.2016.19361. Retrieved 11 February 2016.

10. The Editorial Board (16 February 2016). The Chirp Heard Across the Universe. New York Times. Retrieved 16 February 2016.

11. C. Frajuca, K. L. Ribeiro, O. D. Aguiar, L. A. Andrade, P. J. Castro, N. S. Magalhaes and R. M. Marinho Jr., CQG 21, 2004.

12. C. Frajuca, K. L. Ribeiro, L. A. Andrade, W. F. Velloso Jr., J. L. Melo, O. D. Aguiar, and N. S. Magalhaes, CQG 19, 1961 (2002). 
13. W. W. Johnson and S. M. Merkowitz, Phys. Rev. Lett. 70, 2367 (1993).

14. W. W. Johnson and S. M. Merkowitz, Phys. Rev. D 56, 7513 (1997).

15. S. M. Magalhaes, O. D. Aguiar, W. W. Johnson, and C. Frajuca, Gen. Relat. Grav. 29, 1511 (1997).

16. N. S. Magalhaes, W. W. Johnson, C. Frajuca, and O. D. Aguiar, MNRAS 274, 670 (1995).

17. N. S. Magalhaes, W. W. Jonhson, C. Frajuca, and O. D. Aguiar, Astrophysical Journal 475, 462 (1997). 